\title{
XIMIT - X-Ray Migration Measurement Using Implant Models and Image Templates
}

\author{
Kathrin Burckhardt and Gábor Székely \\ Computer Vision Laboratory, \\ Swiss Federal Institute of Technology, ETH Zürich, \\ [tribur|szekely]@vision.ee.ethz.ch \\ http://www.vision.ee.ethz.ch
}

\begin{abstract}
XIMIT is a newly developed method for precisely measuring the $2 \mathrm{D}$ migration of a hip socket implant using standard radiographs. 2D migration is the change of the $2 \mathrm{D}$ distance between implant and bone observable in the x-ray image. It is measured in order to judge the fixation of the implant in the bone. The proposed method is based on a carefully designed measurement of the $2 \mathrm{D}$ distance minimizing the influence of different sources of errors. The magnitude of the entering errors is minimized by estimating the implant's $3 \mathrm{D}$ orientation and position using the CAD model of the implant and by involving a state of the art template matching algorithm. According to the first error estimate, the new method makes a migration measurement with a standard deviation of about $0.2 \mathrm{~mm}$ possible.
\end{abstract}

\section{Introduction}

Many people suffer from coxarthrosis, the arthrosis of the hip joint. Often, the replacement of the hip by a bone implant is the only way to relieve the pain. About 400.000 artificial hip joints are implanted every year only in Europe. However, despite its success, hip replacement still involves complications. One major problem is the loosening of the cup, the acetabular part of the implant. The fixation of the cup in the bone depends on the design of the implant and on the implantation technique. If a certain kind of replacement has a high tendency to loosen, its extensive use on patients should be prevented. Therefore, the aim is to promptly and reliably find out the fixation properties of a certain cup or implantation technique.

Migration of the implant, which means the change of the cup's position in the bone, is interpreted as an objective sign for loosening. Especially early postoperative migration is supposed to give information about the (later) fixation of the implant in the bone $[6,10,8]$. In [10], it is recapitulated that the risk of a failed ingrowth is minimal if the migration is less than $1 \mathrm{~mm}$ and occurs only in the first year after surgery.

The various methods for measuring the migration can be classified into one $3 \mathrm{D}$ method and several 2D methods. The migration is assessed using time series of x-ray images, divided into reference image(s) and compare images. The 
methods are based on the observation of the change of the distance between the implant and certain bone-related landmarks over time. In the 3D method, RSA (Roentgen Stereophotogrammetric Analysis) [12, 6], all three components of this distance are determined. This is achieved by inserting about 7 metal markers into the bone and into the implant and simultaneously taking two radiographs from two different directions of the pelvis as well as of a calibration frame.

In the $2 \mathrm{D}$ methods, e.g. $[11,3,13,7]$, only the $2 \mathrm{D}$ distance, i.e. the components of the bone-implant distance parallel to the film plane, is observed. The 2D distance is measured in one single x-ray image, by determining a cup reference point and certain bone reference points or lines defined by bony structures. The used x-ray images are the standard radiographs of the hip replacement's follow-up study. The bony structures and the cup reference point are marked by hand using a pencil (in case of conventional radiographs) or by mouse-clicking on digitized radiographs. The magnification of the radiographic system is taken into consideration, either through the division of the $2 \mathrm{D}$ distance by the estimated magnification factor or through the reconstruction of the spatial situation at exposure [7]. In both cases the known radius of the head of the implant's femoral part and the measured size of this radius in the image are used. In [11], the $2 \mathrm{D}$ distance is corrected for pelvis rotation between the exposures by interpreting the varying distance between two bony structures in the x-ray images. In [7], the rotation is taken into consideration by evaluating only pairs of radiographs showing the pelvis in a similar orientation. To select these pairs also image distances between bony structures are compared.

In order to be useful for the predictive early postoperative migration, the migration measurement should have a precision lying in the submillimeter range. This is the case for RSA, of which a maximum standard deviation of $0.1 \mathrm{~mm}$ for the migration parallel to the film plane is reported $[9,6]$. However, RSA is not suited for the clinical routine and thus for a large-scale examination because of the necessary implantation of markers and of the expensive technique required. The existing 2D methods, on the other side, do not require special preparation or equipment, but are probably too imprecise. Dickob et al. [3] evaluated radiographs showing a pelvis phantom with varying orientation to test their method and found a standard deviation of the measured migration of about $1.0 \mathrm{~mm}$. Nunn et al. [11] and Sutherland et al. [13] roughly estimated the errors of their methods to be $3.0 \mathrm{~mm}$ and $2.5 \mathrm{~mm}$, respectively. The precision of different $2 \mathrm{D}$ methods was theoretically estimated in [2] by considering all sources of error and by applying the principle of error propagation. The resulting standard deviations for the two components of the migration in [7], [11], [3] and [13] were found to lie in the range of $0.7 \mathrm{~mm}$ to $2.7 \mathrm{~mm}$.

Measuring the migration using single standard radiographs is affected by the following well known sources of error: the variability of the extrinsic imaging parameters (here the position and orientation of the patient's pelvis at exposure), the unknown intrinsic parameters (here only the film-focus distance), and the lack of definite reference points in the image (here the implant reference point and bony landmarks). It is clear that, because of these sources of errors, it 
will hardly be possible to reach the precision of a stereo radiographic method using implanted markers. Still, 2D migration measurement can be improved. With XIMIT, its optimization is pursued in order to attain a method making the submillimeter measurement of implant migration under clinical conditions possible.

\section{Minimizing the Error of 2D Migration Measurement}

The method proposed here is based on two pillars: On the one side the design of a distance measurement using single radiographs which is as insensitive as possible towards the above mentioned sources of errors (Sect.2.1). On the other side the reduction of the magnitude of the remaining relevant entering errors (Sect. 2.2). The latter is achieved by involving a 2D template matching algorithm to localize the bony structures and by using the known $3 \mathrm{D}$ model of the implant to determine its position and orientation at exposure (thus the acronym $\mathbf{X}$-ray migration measurement using Implant Models and Image Templates).

\subsection{Minimizing the Sensitivity towards the Sources of Error}

Applying the principle of error propagation to different 2D methods for measuring migration, the partial derivatives of the measured $2 \mathrm{D}$ distance with respect to the sources of error were calculated [2]. These derivatives are measures for the significance of the different sources of error for the total precision. They depend on the design of the method, that means on the bony structures which are used, on the definition of the bone reference points or lines, etc. According to the conclusions in [2], the following general rules can be stated:

1. The sensitivity of the measured migration towards pelvis rotation between two exposures can be reduced by choosing bony landmarks with a small 3D distance (especially in sagittal direction) to the cup reference point.

2. The smaller the sagittal component of this $3 \mathrm{D}$ distance, the lower is the influence of the pelvis translation relative to the focus (i.e. the x-ray source).

3. Pelvis rotation around the sagittal axis can be neglected, if a bone reference system defined by two bony landmarks is used.

4. If the correction for magnification is considered for each x-ray image separately, the variability of the focus-film and of the focus-pelvis distance plays no role, and the influence of the rotation is reduced.

The design of the proposed method is based on these observations and on the existing migration measurement methods. It is illustrated in Fig. 1. Considering the Rules 1 and 2, the so-called teardrop figures were chosen as bony landmarks $\left(\mathbf{m}_{1}\right.$ and $\left.\mathbf{m}_{2}\right)$. These figures are the projections of bony structures close to the acetabulum and with a similar sagittal position as its center. The cup reference point (c) is chosen be the cup center. The distance between cup and bone is represented in a coordinate system $\left(\mathbf{e}_{x}, \mathbf{e}_{y}\right)$, which is defined by the line connecting the most caudal points of the teardrop figures and by the 
perpendicular of this line. Thus, the distance is not affected by rotation around the sagittal axis according to Observation 3. Taking Rule 4 into consideration, the measured distance is corrected for magnification through division by the magnification factor $g$, which is estimated for each exposure. The magnification factor corresponds to the ratio between focus-film and focus-implant distance and is determined as described in Sect. 2.2. The measured 2D distance indicated with $\mathbf{d}$ is given by

$$
\mathbf{d}=\frac{1}{g}\left(\begin{array}{l}
\left(\mathbf{c}-\mathbf{m}_{1}\right) \cdot \mathbf{e}_{x} \\
\left(\mathbf{c}-\mathbf{m}_{1}\right) \cdot \mathbf{e}_{y}
\end{array}\right) .
$$

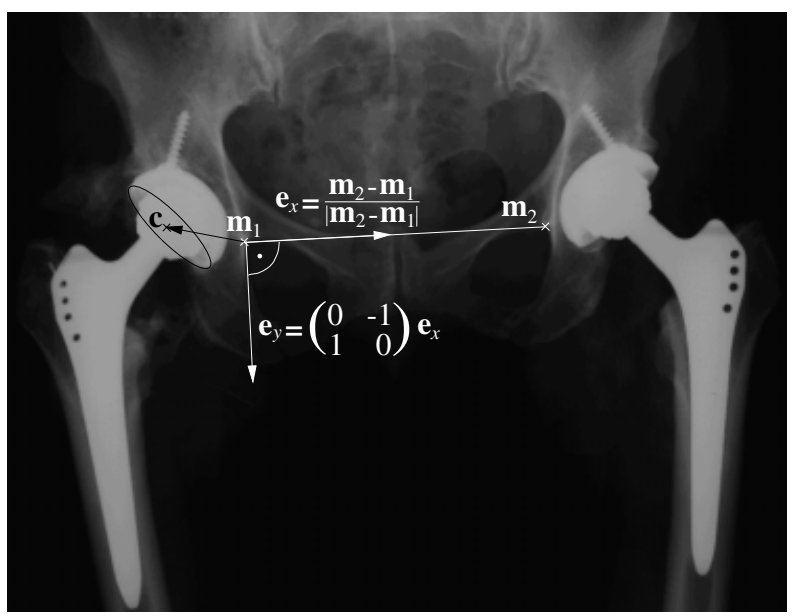

Fig. 1. The measured 2D distance in XIMIT

\subsection{Minimizing the Magnitude of the Entering Errors}

Using the 3D Model of the Implant. In order to precisely determine the image position of the cup and its distance to the focus, its 3D position is determined using its CAD model and the approximate focus-film constellation at exposure. The principle of "Analysis-by-Synthesis" is applied: the implant's position and orientation parameters are estimated by minimizing the difference between a synthesized and the original x-ray image of the cup.

The focus-film constellation is defined by the intrinsic parameters, which are the focus-film distance $f$ and the intersection of the central beam with the film $\mathbf{t}$ (see Fig. 2). $f$ is assumed to have the nominal value of the $\mathbf{x}$-ray unit, and $\mathbf{t}$ is estimated to be the film center, because in the clinical practice the central beam is directed to this point. 
In this focus-film system, the $3 \mathrm{D}$ orientation and position of the implant is first roughly estimated by the manual fitting of an ellipse to the circumference of the cup's equatorial plane. The ellipse is shown in Fig. 1. With this initial estimate the Analysis-by-Synthesis algorithm is started, searching the cup's 3D orientation and position where the difference between the synthetic and the original x-ray image of the cup is at minimum. The difference measure, chosen to be the sum of the squared intensity differences at each pixel, is minimized using a combined modified Newton and Gauss-Newton algorithm. The x-ray image is synthesized by calculating the absorption of the x-rays reaching from the assumed position of the focus to each pixel. The absorption depends on the x-rays' lengths inside the cup, which are gained by intersecting each ray with the cup's surface given by the CAD model.

To get the image position $\mathbf{c}$ of the cup center, the estimated 3D cup center is projected on the x-ray film plane using the same intrinsic parameters as used for Analysis-by-Synthesis. The magnification factor $g$ is yielded by division of the film-focus distance by the estimated cup's $z$-position. Even if the assumed intrinsic parameters differ from the real ones at exposure, $\mathbf{c}$ and $g$ are precisely determined, because the difference is automatically neutralized by an appropriate $x$ - $y$ - and $z$-translation of the cup during Analysis-by-Synthesis.

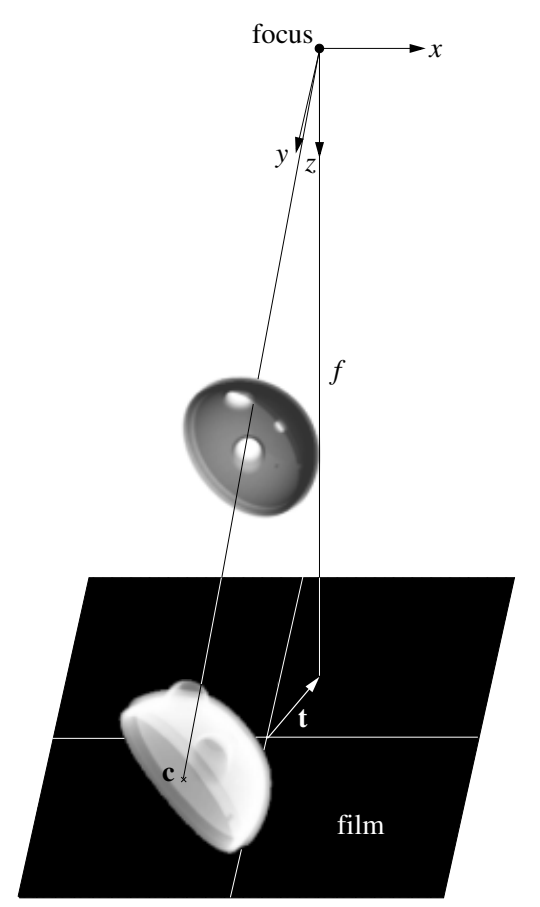

Fig. 2. Illustration of the spatial situation while synthesizing the cup's x-ray image 
Locating the Bony Landmarks by Template Matching. Template matching is a general procedure to estimate the mapping between a template, i.e. a structured area in a reference image, and a patch, i.e. the corresponding area in a compare image. The mapping is expressed by a geometrical transformation (translation, rotation, scale, etc.) in the patch's space. The translational part of this transformation can be used to locate the patch in the compare image. In XIMIT, a least square matching (LSM) $[1,4]$ is applied, which means that the transformation parameters are estimated through a least square minimization.

Here, the template is the area containing a teardrop figure in a reference $\mathrm{x}$ ray image. The patch is the area of the same teardrop figure in a compare image. The transformation working best is a translation plus rotation to compensate for rotation of pelvis around the sagittal axis. It is represented by $\mathbf{x}_{i}^{\prime}=\mathbf{R}_{\mathrm{LSM}} \mathbf{x}_{i}+$ $\mathbf{t}_{\mathrm{LSM}}$, where $\mathbf{x}_{i}$ and $\mathbf{x}_{i}^{\prime}$ indicate the original and the transformed coordinates of pixel i, $\mathbf{t}_{\mathrm{LSM}}$ the translation, and $\mathbf{R}_{\mathrm{LSM}}$ the rotation matrix. The transformation's parameters are found by minimizing the sum of the squared differences between the intensities of the template at $\mathbf{x}_{i}$ and of the patch at $\mathbf{x}_{i}^{\prime}$.

The template is manually defined by marking in the reference radiograph the area of the teardrop figures which is relatively independent from the pelvis orientation. In the reference as well as in the compare x-ray image, the most caudal points of the teardrop figures are marked (see points in Fig. 3a and Fig. 3b). Subtracting the points and setting $\mathbf{R}_{\mathrm{LSM}}=\mathbf{I}$ gives the initial estimate for LSM. For the evaluation of all compare images belonging to a reference image, the once marked points of the reference teardrop figures are kept. Adding to these points the translational parts $\mathbf{t}_{\mathrm{LSM}}$ of the resulting affine transformations gives the points' positions $\mathbf{m}_{1,2}$ in the compare images.

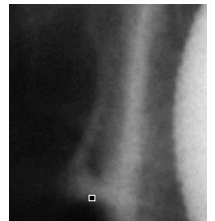

Fig. 3a

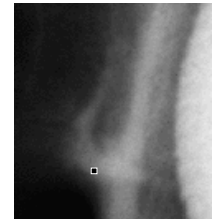

Fig. 3b

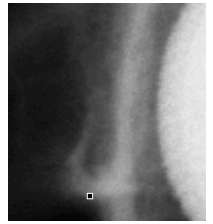

Fig. 3c

Fig. 3. The marked teardrop figure in the reference and in the compare x-ray image (Fig. $3 \mathrm{a}$ and $3 \mathrm{~b}$ ), and the compare image warped with the transformation estimated using LSM (Fig. 3c).

\section{$3 \quad$ Tests and Results}

\subsection{Theoretically Estimated Precision}

The scheme of theoretically estimating the standard deviation worked out in [2] was applied to (1). The following sums of error components resulted, in which 
the coefficients are the partial derivatives of $\mathbf{d}$ with respect to the variables representing the sources of error:

$$
\begin{aligned}
\sigma_{d_{x}}^{2}= & \left(0.01806 \sigma_{\delta \alpha}\right)^{2}+\left(-0.00098 \sigma_{\delta \beta}\right)^{2}+\left(-0.00277 \sigma_{\delta \gamma}\right)^{2}+\left(0.00264 \sigma_{\delta T_{x}}\right)^{2}+\left(-0.00020 \sigma_{\delta T_{z}}\right)^{2}+ \\
& \left(0.79091 \sigma_{\delta c_{x}}\right)^{2}+\left(-0.79091 \sigma_{\delta m_{1 x}}\right)^{2}+\left(-0.08648 \sigma_{\delta m_{1 y}}\right)^{2}+\left(0.08648 \sigma_{\delta m_{2 y}}\right)^{2}+ \\
& \left(-26.47220 \sigma_{\delta g}\right)^{2}+\left(-0.00020 \sigma_{\delta f}\right)^{2} \\
\sigma_{d_{y}}^{2}= & \left(-0.04433 \sigma_{\delta \alpha}\right)^{2}+\left(-0.00264 \sigma_{\delta T_{y}}\right)^{2}+\left(-0.00001 \sigma_{\delta T_{z}}\right)^{2}+ \\
& \left(-0.79091 \sigma_{\delta c_{y}}\right)^{2}+\left(0.99603 \sigma_{\delta m_{1 y}}\right)^{2}+\left(-0.20512 \sigma_{\delta m_{2 y}}\right)^{2}+\left(-11.16060 \sigma_{\delta g}\right)^{2}+\left(-0.00001 \sigma_{\delta f}\right)^{2}
\end{aligned}
$$

With the same notation as in $[2], \sigma_{\delta \alpha, \delta \beta, \delta \gamma}, \sigma_{\delta T_{x, y, z}}$, and $\sigma_{\delta f}$ indicate the standard deviation of the pelvis orientation ${ }^{1}$, of its position, and of the film-focus distance, respectively. The error in locating the cup center and the teardrop figures and the one in determining the correction for magnification are represented by $\sigma_{\delta c_{x, y}}$, $\sigma_{\delta m_{1,2 x, y}}$, and $\sigma_{\delta g}$.

For comparison of the theoretical estimate with the experimental results, $\sigma_{\delta T_{x, y}}$ and $\sigma_{\delta \alpha, \delta \beta}$ is derived using the pelvis position and orientation parameters in the validation exposures (see next section). Calculating the standard deviations of these parameters listed in the middle column of Table 1 yields $\sigma_{\delta T_{x}}=22 \mathrm{~mm}, \sigma_{\delta T_{y}}=27 \mathrm{~mm}, \sigma_{\delta \alpha}=4.1^{\circ}$, and $\sigma_{\delta \beta}=2.3^{\circ}$. From [2], the values $\sigma_{\delta \gamma}=3.0^{\circ}, \sigma_{\delta T_{z}}=15 \mathrm{~mm}$, and $\sigma_{\delta f}=100 \mathrm{~mm}$ were adopted. LSM and Analysis-by-Synthesis were run ten times with a new definition of the template and of the initial estimates. The standard deviations $\sigma_{\delta m_{1,2 x, y}}=0.07 \mathrm{~mm}$ and $\sigma_{\delta c_{x, y}}=0.015 \mathrm{~mm}$, and $\sigma_{\delta g}=0.0008$ were derived from these tests made using several radiographs of two different pelves.

Regarding the coefficients in (2) and considering the size of these standard deviations, the sources of error having a significant influence are: the pelvis rotation around the $x$-axis, the error in locating the cup and the bony landmarks, the error of the correction for magnification, and less importantly also the variable pelvis $x$-y-position. Inserting these standard deviations in $(2)$, the theoretical error of the measured $2 \mathrm{D}$ distance is: $\sigma_{d_{x}}=0.11 \mathrm{~mm}, \sigma_{d_{y}}=0.21 \mathrm{~mm}$.

\subsection{Experimental Validation}

XIMIT was validated using x-ray images of a pelvis phantom equipped with a cup and mounted on a cage. The phantom-cage system allows the simulation of implant migration in the bone and of pelvis translation in the film-focus system with a precision of about $0.05 \mathrm{~mm}$. It also makes the rotation of the pelvis with a precision of about $0.05^{\circ}$ possible.

In the first experiment, the pelvis was translated along the $x$ - as well as along the $y$-axis, keeping the cup-bone distance constant. Then, the orientation of the pelvis was varied by rotation, together with the cup, around these two axes. Finally, exposures were made at different cup distances to the bone, keeping the

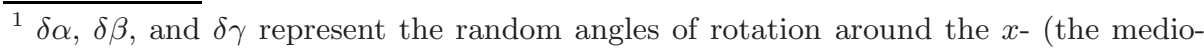
lateral), the $y$ - (the cranio-caudal), and the $z$ - (the sagittal) axis
} 
position and orientation of the pelvis constant. The radiographs were scanned with a pixel resolution of $300 \mathrm{dpi}$ and a gray value resolution of $12 \mathrm{bit}$.

In the Tables 1 and 2, the measured migration values are listed, i.e. the differences $\Delta \mathbf{d}=\left(\Delta d_{x}, \Delta d_{y}\right)$ between the bone-implant distance $\mathbf{d}$ measured in the reference radiograph and in the ones made under changed conditions. Their standard deviations from groundtruth are also shown.

The standard deviations in the tables mainly agree with the theoretical error estimate: From (2), similar values result, if the standard deviations $\sigma_{\delta \alpha}, \sigma_{\delta \beta}$, $\sigma_{\delta T_{x}}$, or $\sigma_{\delta T_{y}}$ corresponding to the unchanged position or orientation parameters are set to zero. Only the errors of $d_{x}$ at a $x$-translation and especially the one at a rotation around the $y$-axis significantly differ from the theoretical result, because these motions influenced the shape of the teardrop figures. The values in Table 2 show that the measured 2D distance change in fact corresponds to the projection of the implant migration on the $x-y$-plane.

Table 1. Measured migration while translating and rotating the pelvis

\begin{tabular}{|c|c|c|c|}
\hline & pelvis motion & $\Delta d_{x}[\mathrm{~mm}]$ & $\Delta d_{y}[\mathrm{~mm}]$ \\
\hline groundtruth: no migration & & 0.0 & 0.0 \\
\hline & translation in $x$-direction & & \\
\hline & $-2.4 \mathrm{~cm}$ & 0.37 & 0.01 \\
\hline & $-1.2 \mathrm{~cm}$ & 0.19 & -0.13 \\
\hline & $1.2 \mathrm{~cm}$ & -0.15 & -0.06 \\
\hline & $2.4 \mathrm{~cm}$ & -0.16 & -0.05 \\
\hline \multirow[t]{6}{*}{ standard deviation } & & 0.26 & 0.05 \\
\hline & translation in $y$-direction & & \\
\hline & $-3.0 \mathrm{~cm}$ & 0.01 & -0.11 \\
\hline & $-1.5 \mathrm{~cm}$ & 0.01 & -0.07 \\
\hline & $1.5 \mathrm{~cm}$ & 0.06 & -0.01 \\
\hline & $3.0 \mathrm{~cm}$ & 0.03 & 0.06 \\
\hline \multirow[t]{7}{*}{ standard deviation } & & 0.03 & 0.07 \\
\hline & rotation around $x$-axis & & \\
\hline & $-4.39^{\circ}$ & -0.00 & -0.34 \\
\hline & $-2.19^{\circ}$ & -0.09 & -0.08 \\
\hline & $2.19^{\circ}$ & 0.21 & -0.04 \\
\hline & $3.29^{\circ}$ & 0.04 & 0.33 \\
\hline & $5.47^{\circ}$ & 0.32 & 0.03 \\
\hline \multirow[t]{7}{*}{ standard deviation } & & 0.16 & 0.24 \\
\hline & rotation around $y$-axis & & \\
\hline & $-2.19^{\circ}$ & 0.41 & 0.06 \\
\hline & $-1.09^{\circ}$ & 0.16 & -0.06 \\
\hline & $1.09^{\circ}$ & -0.18 & -0.02 \\
\hline & $2.19^{\circ}$ & -0.20 & -0.01 \\
\hline & $3.29^{\circ}$ & -0.48 & 0.04 \\
\hline standard deviation & & 0.35 & 0.05 \\
\hline
\end{tabular}


Table 2. Measured migration at constant position and orientation of pelvis

\begin{tabular}{ccccc} 
& groundtruth & \multicolumn{2}{c}{ measurement } \\
$\Delta d_{x}[\mathrm{~mm}]$ & $\Delta d_{y}[\mathrm{~mm}]$ & $\Delta d_{x}[\mathrm{~mm}]$ & $\Delta d_{y}[\mathrm{~mm}]$ \\
\hline 0.0 & 0.0 & -0.13 & 0.00 \\
0.0 & 0.0 & 0.02 & -0.13 \\
0.0 & 0.0 & -0.04 & -0.08 \\
0.60 & 0.72 & 0.52 & 0.78 \\
1.20 & 1.44 & 1.08 & 1.57 \\
& 1.80 & 2.17 & 1.82 & 2.12 \\
standard deviation & & & 0.09 & 0.07
\end{tabular}

\section{Conclusions}

According to the above results, XIMIT substantially improves implant migration measurement using standard radiographs. It makes a 2D migration measurement of artificial hip sockets in the submillimeter range possible. However, achieving this precision requires the restriction of pelvis rotation between two exposures to about $\pm 3^{\circ}$ and $\pm 2^{\circ}$ around the medio-lateral and the cranio-caudal axis, respectively.

This limitation is necessary mainly because pelvis rotation alters the bony reference structures in the image, i.e. the most caudal points of the teardrop figures. At a variable orientation, the exact identification of these bony landmarks in all images is difficult. Another problem of natural bony landmarks may be the interobserver variability of their location in the image, which is not tested up to the present. Despite its automation using a template matching algorithm, the location may be influenced by the user as it depends on the definition of the template. Because of these two reasons, implanted markers as bone reference points instead of natural bony landmarks would improve XIMIT.

The pelvis rotation between two exposures may be restricted by putting a pad under the patients knees, by repeating exposures not comparable to the preceding ones, or by omitting for evaluation the follow-up series' radiographs which show the pelvis in an extraordinary orientation. These possibilities require a measure for pelvis rotation. Additional studies are planned to find such a measure by evaluating the appearance of certain bony structures. For example, the alteration at certain rotation angles of the position of the coccyx relative to the symphysis pubis or of the shape of the foramina obturatoria can be investigated. Different from the pelvis bone, the orientation of the implant is automatically estimated in XIMIT by applying Analysis-by-Synthesis. Therefore, having a quantitive measure for pelvis orientation also the rotation of the cup relative to the bone, intended to be measured already in [5], could be determined.

The proposed method is well suited for the measurement of implant migration integrated in the clinical routine. It makes reliable large-scale examinations of the 
fixation properties of artificial hip sockets possible. At the same time the basic algorithms can be well generalized to other anatomical regions and provide a good approach for migration measurement, for example, of knee or of shoulder implants.

\section{Acknowledgment}

This work has been supported by the grant No. 9803 of the Synos Foundation, Münsingen-Bern, Switzerland

\section{References}

[1] M. Berger. The framework of least squares template matching. Technical Report 180, Communication Technology Lab, Computer Vision Group, ETH Zürich, 1998.

[2] K. Burckhardt, Ch. Brechbühler, Ch. Gerber, J. Hodler, and Gabor Székely. Precision of distance determination using $3 \mathrm{~d}$ to $2 \mathrm{~d}$ projections: The error of migration measurement using x-ray images. MedIA, in press.

[3] M. Dickob, J. Bleher, and W. Puhl. Standardisierte Pfannenwanderungsanalyse in der Hüftendoprothetik mittels digitaler Bildverarbeitung. Unfallchirurg, 97:92-97, 1994.

[4] A Grün. Adaptive least squares correlation: A powerful image matching technique. South African Journal of Photogrammetry, Remote Sensing 8 Cartography, 14(3):175-187, 1985.

[5] B. Jamaraz, C. Nikou, T.J. Levison, A.M. DiGioia III, and R.S. LaBarca. CupAlign: Computer-Assisted Postoperative Radiographic Measurement of Acetabular Components Following Total Hip Arthroplasty. In Proc. of MICCAI, 1999.

[6] J. Kärrholm, P. Herberts, P. Hultmark, H. Malchau, B. Nivbrant, and J. Thanner. Radiostereometry of Hip Prostheses: Review of Methodology and Clinical Results. Clin Orthop, November(344):94-110, 1997.

[7] M. Krismer, R. Bauer, J. Tschupik, and P. Mayrhofer. EBRA: a method to measure migration of acetabular components. J Biomechanics, 28:1225-1236, 1995.

[8] M. Krismer, B. Stöckl, M. Fischer, R. Bauer, P. Mayrhofer, and M. Ogon. Early migration predicts late aseptic failure of hip sockets. J Bone Joint Surg, 78-B426, 1996.

[9] B. Mjöberg, G. Selvik, L.I. Hansson, R. Rosenqvist, and R. Önnerfält. Mechanical loosening of total hip prostheses: A radiographic and roentgen stereophotogrammetric study. J Bone Joint Surg, 68-B:646-652, 1986.

[10] K.G. Nilsson and J. Kärrholm. RSA in the assessment of aseptic loosening. $J$ Bone Joint Surg [Br], 78-B(1):1-2, 1996.

[11] D. Nunn, M.A.R. Freeman, P.F. Hill, and S.J.W. Evans. The measurement of migration of the acetabular component of hip prosthesis. J Bone Joint Surg [Br], 71:629-631, 1989.

[12] G. Selvik. Roentgen Stereophotogrammetric Analysis. Acta Radiologica, 31:113$126,1990$.

[13] C.J. Sutherland, A.H. Wilde, L.S. Borden, and K.E. Marks. A Ten-Year Follow-up of One Hundred Consecutive Müller Curved-Stem Total Hip-Replacement Arthroplasties. J Bone Joint Surg, 64-A(7):970-982, 1982. 